\title{
Effects of Velocity Mismatch and Transit Time on Linearized Electro-Optic Modulators
}

\author{
Uri V. Cummings and William B. Bridges \\ California Institute of Technology \\ Pasadena California
}

\begin{abstract}
Gain, noise figure, and dynamic range limited by intermodulation and harmonic distortion were calculated for simple analog optical links employing different linearized electro-optic modulators with a representative set of link parameters in Ref.[1]. However, that analysis ignored the effects of transit time and velocity mismatch. Since linearized modulators typically involve critical cancellations to reduce distortion, it is important to determine how transit-time and velocity mismatch affect the linearization.
\end{abstract}

We have used a numerical algorithm introduced by Farwell and Chang [2] to analyze the same links as in [1] with additional representative values of velocity mismatch (microwave velocity $=$ c/4.0, optical velocity $=c / 2.2$, typical of a transmission line on lithium niobate) and a modulator length of $10 \mathrm{~mm}$. As in [2], the modulator structure is divided into many short segments, with a matrix written for each segment. The modulating voltage is assumed uniform in magnitude and phase over the segment, but the phase of successive segments is shifted by an amount appropriate to the velocity mismatch. The program then multiplies all the matrices (typically 128) to relate the output to input. Voltages at two closely-space microwave frequencies $f_{1}$ and $f_{2}$ are applied and the output FFT-analyzed to find the amplitudes of the signal $\left(f_{1}\right)$, second harmonic $\left(2 f_{1}\right)$, and intermodulation $\left(2 f_{1}-f_{2}\right)$. The dynamic range (broad band) is determined by finding the input power that makes the intermodulation distortion (IMD) or the second harmonic component equal to the noise level. If only a sub-octive bandwidth link is required, then the dynamic range (narrow band) is limited by the IMD alone.

The results for worst-case velocity mismatch are shown in Fig. 1, which compares the dynamic ranges for four different modulators (see ref. 1 for modulator and link details): the simple directional coupler modulator biased at its minimum second harmonic point (43\% of the transfer voltage) [DCM.43], the same DCM, but biased at its maximum dynamic range point (79\% of the transfer voltage) [DCM.79], the directional coupler with two added d-c sections for linearization, from ref. [2], referred to here as the UCSD modulator, considered as limited by intermodulation distortion alone [UCSDw/o2] or limited by intermodulation or second harmonic, whichever is worse [UCSDw2]. In the case of the DCM.43, the limitation switches from IMD below $1 \mathrm{GHz}$ to second harmonic above. For DCM.79, the second harmonic is so bad even at very low frequencies, that only the IMD limited (narrow band) dynamic range is shown. Figure 2 shows the gain degradation with frequency for these same three modulators, plus the simple Mach-Zehnder for reference. As suggested by Fanwell and Chang, the calculation scales as $f_{1} * L * \Delta n$, with $L=10 \mathrm{~mm}$ and $\Delta n=1.8$ for our calculations, so these curves apply as well to partially velocitymatched modulators [e.g., ref. 3] by re-scaling the abscissa to the appropriate length and residual velocity mismatch.

The curves in Figs 1 and 2 show that the second harmonic severely decreases the dynamic range of the UCSD modulator at just a few hundred $\mathrm{MHz}$. The simple DCM dynamic ranges are only slightly less rapidly decreased. We conclude that velocity matching is required to preserve linearization at much lower frequencies than would be required to simply preserve gain.

The program written for this study also easily allows calculations for periodically-rephased modulators $[e .9 ., 4,5]$ since the modulator is already broken up into a series of incremental matrices. Thus if the modulator is allowed to be mismatched for a few matrices and then rephased for the next few and so on, we have the results shown in Fig. 3 for a four segment modulator ( 3 rephasings). The same three modulators are considered. With rephasing, the DCM.43 dynamic range is flat to beyond $30 \mathrm{GHz}$ at its d-c value, limited only by IMD, not second harmonic. The DCM.79 (IMD-limited, but with terrible second harmonic) is also greatly improved. The UCSD modulator dynamic range drops off less rapidly, but still seriously. Rephasing more often improves all modulators, as shown in Table 1. 
We have also added electrode loss to the calculation (requiring only an exponential decrease in amplitude from matrix to matrix), and have compared the effect of loss on perfectly velocity matched modulators. It appears that loss alone reduces the dynamic range of linearized modulators by upsetting the critical distortion-canceling conditions. The effects of loss are less destructive in the rephased modulators, since each segment is "re-amplituded" as well as rephased.

This work was supported by USAF Rome Laboratory under contract F30602-92-C-0005, N.P. Bernstein, program manager. The authors wish to thank J. H. Schaffner for many technical discussions.

[1] W. B. Bridges and J. H. Schaffner, "Distortion in Linearized Electro-Optic Modulators," IEEE Trans. On Microwave Theory and Tech., Vol. 43, pp2184-2197

[2] M. L. Farwell and W. S. C. Chang, "Simulating the Response of Coupled Channel and Interferometric Modulator Designs," J. Lightwave Tech., Vol. 13, pp 2059-2068, October 1995.

[3] G. K. Gopalakrishna, C. H. Bulmer, W. K. Burns, and R. W. McElhanon, " 40GHz Low Half-Wave Voltage Ti:LiNbO ${ }_{3}$ Intensity Modulator," Elect. Lett., Vol. 28, pp 826-827, April 23, 1992.

[4] J. H. Schaffner and W. B. Bridges, "Broad Band, Low Power Electro-Optic Modulators Apparatus and Method with Segmented Electrodes," U. S. Patent 5,291,565, September 1994.

[5] W. B. Bridges, F. T. Sheehy and J. H. Schaffner, "Wave-Coupled Electrooptic Modulator for Microwave and Millimeter-Wave Modulation," Photon. Tech. Lett. Vol. 3, pp 133-135, February 1991.
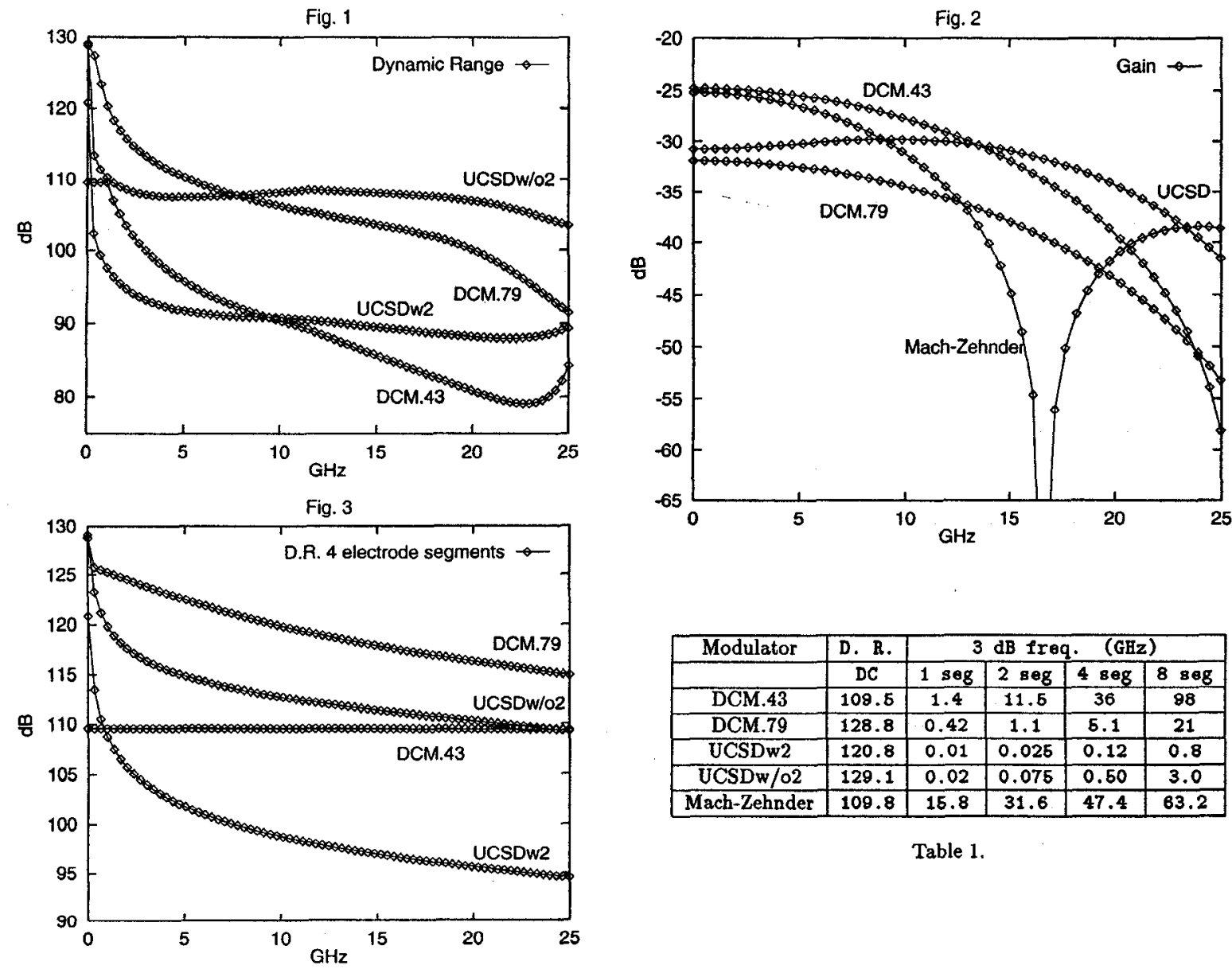

\begin{tabular}{|c|c|c|c|c|c|}
\hline Modulator & D. R. & \multicolumn{3}{|c|}{3 dB freq. (GHz) } \\
\hline & DC & 1 seg & $2 \mathrm{seg}$ & $4 \mathrm{seg}$ & $8 \mathrm{seg}$ \\
\hline DCM.43 & 109.5 & 1.4 & 11.6 & 36 & 98 \\
\hline DCM.79 & 128.8 & 0.42 & 1.1 & 5.1 & 21 \\
\hline UCSDw2 & 120.8 & 0.01 & 0.025 & 0.12 & 0.8 \\
\hline UCSDw/o2 & 129.1 & 0.02 & 0.075 & 0.50 & 3.0 \\
\hline Mach-Zehnder & 109.8 & 15.8 & 31.6 & 47.4 & 63.2 \\
\hline
\end{tabular}

Table 1. 